Artigo recebido em 26.11.2018 / Aprovado em 05.01.2019

\title{
ARBITRAGEM COMO MEIO DE SOLUÇÃO DE CONFLITOS ENVOLVENDO A TECNOLOGIA BLOBKCHAIN E SMART CONTRACTS
}

\section{ARBITRATION AS A MEANS OF CONFLICT SOLUTION INVOLVING BLOBKCHAIN AND SMART CONTRACTS TECHNOLOGY}

\author{
Benjamim Siqueira de Amorim ${ }^{1}$
}

Alessandro Jacomini ${ }^{2}$

\section{RESUMO}

O presente artigo objetivou demonstrar como a arbitragem tem o potencial de ser um meio adequado para lidar com conflitos envolvendo a tecnologia blockchain. Para tanto, num primeiro momento, buscou-se delinear os aspectos fundamentais desta nova tecnologia, as mudanças que propõe este fenômeno, destacando-se, dentre suas diversas aplicações, o uso dos smart contracts, analisando-se a sua diferença em relação aos contratos tradicionais, em especial a seus possíveis conflitos que possuem características próprias. É por esse prisma que se desejou analisar as vantagens atuais do uso da arbitragem, e como elas se adequam para lidar com estes específicos litígios. Trata-se de uma abordagem cujo objeto, isto é, a blockchain, ainda está em fase inicial de usos, desenvolvimentos e aplicações, assim, não possui o presente estudo a pretensão de respostas inteiramente conclusivas. A metodologia empregada foi a dialética, com abordagem qualitativa e propósito descritivo, incluindo o procedimento de levantamento bibliográfico, leituras de artigos e monografias.

Palavras-chave: Blockchain; Contratos Inteligentes; Arbitragem.

\section{ABSTRACT}

The present article aimed to demonstrate how arbitration has the potential to be an adequate means to deal with conflicts involving blockchain technology. In order to do so, the first step was to outline the fundamental aspects of this new technology, the changes proposed by this phenomenon, highlighting, among its various applications, the

\footnotetext{
${ }^{1}$ Graduando em Direito pelo Centro Universitário Adventista de São Paulo/ UNASP - campus Engenheiro Coelho. Escrevente técnico judiciário do Tribunal de Justiça de São Paulo. E-mail: siqueiranomade@gmail.com

2 Doutor em Direito pela Pontifícia Universidade Católica de São Paulo. Professor do Curso de Direito do Centro Universitário Adventista de São Paulo / UNASP - campus Engenheiro Coelho. E-mail: alessandro.jacomini@unasp.edu.br
} 
use of smart contracts, analyzing their difference in relation to contracts traditional, in particular their possible conflicts that have their own characteristics. It is from this perspective that one wished to analyze the current advantages of using arbitration, and how they fit to deal with these specific litigations. It is an approach whose object, that is, the blockchain, is still in the initial phase of uses, developments and applications, so the present study does not have the pretension of entirely conclusive answers.

Keywords: Blockchain; Smart Contracts; Arbitration.

\section{CONSIDERAÇÕES INICIAIS.}

A revolução por parte da informática trouxe profundas modificações em todos os setores da vida humana. Direito, economia e até mesmo a organização da sociedade vive hoje uma fase de grandes transições.

Neste contexto, uma das novidades mais interessantes diz respeito à evolução dos sistemas de pagamentos, bem como o modo de realizar contratos, ambos ligados a novas oportunidades oferecidas pela tecnologia blockchain. Dentre outras, esta tecnologia possui a característica de não depender de uma autoridade central para sua execução, de reduzir custos de transações, de limitar possíveis violações de propriedades, bem como a não modificação unilateral por parte do usuário.

Precisamente, as características e formas de operação dessa tecnologia em suas diversas aplicações, adicionado ao seu desenvolvimento e aceitação mundial entre comerciantes e indústrias, compeliram ao surgimento de conflitos e questões legais importantes que não podem ser ignorados no mundo do Direito.

Os litígios advindos de tal tecnologia possuem características peculiares, devendo-se considerar alguns pontos controvertidos, como por exemplo, de que maneira tais disputas merecem ser gerenciadas quando não há, ao certo, um sistema legal que corresponde às mesmas. De fato, não existe certeza de como se dará a resolução de conflitos oriundos da tecnologia blockchain nas suas variadas aplicações, em especial com os smart contract - um modo diferente de se realizar contratos.

Com a utilização do método dialético, o objetivo do presente artigo é abordar a arbitragem como um dos meios mais adequado de resolução de tais litígios. Em tal metodologia foi utilizada a abordagem qualitativa e propósito descritivo, incluindo o 
procedimento de levantamento bibliográfico, leituras de artigos e monografias, adentrando-se à análise do fenômeno da blockchain, apresentando suas peculiaridades e importantes transformações trazidas em algumas esferas de negócios. Isso leva, por conseguinte, à análise de seu uso na formação dos contratos inteligentes, trazendo à tona algumas situações em que este pode gerar conflitos incomuns.

Por fim, a conclusão faz uma análise prospectora da tecnologia utilizada no bloco, o papel da arbitragem em seu desenvolvimento, assim como a importância de árbitros e operadores do Direito de se aproximarem desta inovação.

\section{BLOCKCHAIN}

O termo "blockchain" representa, nos dias de hoje, a grande revolução que está surgindo na tecnologia da internet. Por sinal, é a tecnologia que está por trás das chamadas criptomoedas, isto é, uma forma de descentralização que utiliza técnicas de criptografia para garantir a segurança dos intercâmbios entre os usuários. No entanto, para muitos, tais termos permanecem uma incógnita.

Para explanar o bloco, necessário se faz mencionar o Bitcoin, a mais importante entre as criptomoedas, cujo nome foi dado como uma nova classe de dinheiro que toma a forma de uma moeda digital. Lançada no ano de 2009, foi criada com o propósito de alcançar maiores vantagens do que aquelas oferecidas pelo sistema financeiro tradicional (LÓPEZ, 2015, P. 44).

Antes, no ano de 2008, foi publicado um paper, tendo como autor Satoshi Nakamoto ${ }^{3}$, com o título "Bitcoin: A Peer-to-Peer Electronic Cash System", em que demonstra-se o funcionamento do Bitcoin. Neste trabalho, o Bitcon é tratado como «a purely peer-to-peer version of eletronic cash would allow online payments to be sent directly from one party to another without going through a financial institution ${ }^{4}$.

De início, pode-se distinguir entre o Bitcoin (B maiúscula) como um protocolo de comunicação, um algoritmo que visa acelerar e assegurar as transações feitas pela

\footnotetext{
${ }^{3}$ Provável pseudônimo, pois ainda não foi revelada a sua identidade.

${ }^{4}$ Cf. Disponível em: <https://bitcoin.org/bitcoin.pdf> Acesso em: 13 de jan. 2018.
} 
internet, e o bitcoin (b minúscula) como um novo tipo de moeda, utilizada principalmente para transações online.

Em relação ao protocolo, a sua intenção é de permitir um envio da moeda de maneira veloz, segura e econômica através da rede, garantindo o anonimato e propondo um modelo descentralizado, isto é, sem a intervenção de qualquer sujeito que controle as transações.

A integridade e a autenticidade das transações são garantidas por criptografia e pela blockchain, um registro público e compartilhado, acessível gratuitamente por cada usuário da rede, em que se baseia o sistema Bitcoin inteiro. Em outras palavras, o bloco executa determinadas funções semelhantes a um livro de registro online, anotando todas as transações realizadas e os usuários que participam da rede (CAPOGNA; PERAINO, 2015, p. 37).

Dentre as inovações trazidas e utilizadas pela criptomoeda em análise, a blockchain é provavelmente a mais importante, pois fornece um banco de dados que inclui todas as transações realizadas na rede Bitcoin ao longo de sua história. Trata-se de uma espécie de registro digital exclusivo, distribuído e disponível publicamente, permanente e resistente a alterações, mantido em funcionamento pela atividade conjunta de todos os nós (comunicação entre clientes) do sistema (CAPOGNA; PERAINO, 2015, p. 36).

Tal feito é realizado pela técnica peer-to-peer, uma arquitetura particular da rede de computadores na qual o computador de um usuário individual dialoga diretamente com os computadores de outros usuários. Os nós são equivalentes uns aos outros, podendo tanto executar funções de clientes como de servidores para todos os outros nós da rede, diferentemente da arquitetura tradicional cliente-servidor, em que a comunicação não ocorre diretamente entre os usuários (ZEN, 2015, p. 37).

No bloco, o registro pode ser público, usados para criptocorrências, ou privado, para serem usados por várias empresas. Entretanto, ambos se direcionam à determinadas transações, distribuindo-as para milhares de participantes em cadeia mundial. Ninguém o controla e cada pessoa na cadeia tem acesso imediato aos dados que os computadores, por meio de algoritmos complexos, convalidam as informações que foram distribuídas. 
A grande vantagem introduzida pela blockchain é a de deixar de impor, de modo geral, a necessidade de um terceiro de confiança para a execução de contratos, como notário/tabelião ou intermediário. A plataforma permite uma execução automatizada e confiada à criptografia, o que permite proteger os participantes dos riscos de não cumprimento de uma das partes (BURRICCO, 2017, p. 27).

Por conseguinte, o bloco acrescenta um componente de maior transparência e eficiência na gestão dos custos, bem como, priva a execução dos contratos de discrição típicas do fator humano. À luz desta série de implicações, ele pode ser uma inovação revolucionária para muitos tipos de contratos e inúmeras atividades comerciais.

A inovação provocada pela estrutura do bloco foi tão importante e funcional que vários indivíduos propuseram usá-la em outras áreas, como políticas, jurídicas, sociais e científicas (BURRICCO, op. cit., loc. cit.).

Em junho de 2018, a Microsoft anunciou um desenvolvimento, junto com o Banco Central Brasileiro, de um sistema que utiliza a blockckain para implementar a troca de informações entre algumas instituições reguladoras e aquele órgão financeiro. Tal parceria visa facilitar a integração de informações entre o Banco Central, a CVM (Comissão de Valores Mobiliários) e a SUSEP (Superintendência de Seguros Privados) que, dispendia demasiado tempo e recursos para troca de dados, sendo por telefonemas, papéis, e-mails, gerando baixa eficiência ${ }^{5}$.

Em setembro de 2018, a JPMorgan Chase \& Co, uma das maiores instituições que presta serviços financeiros, anunciou uma colaboração com 75 bancos de diversos países para utilizar o seu sistema de transações fundamentado na inovação da blockckain. Tal sistema visa acelerar transações de valores entre países com maior segurança 6 .

Em novembro de 2018, no relatório de transição de governo 2018-2019 do presidente eleito daquele ano, há a menção à tecnologia blockckain, inserido no Capítulo intitulado de "Governo Digital", tratando a mencionada tecnologia como um protocolo de confiança "a ser utilizado para autenticar transações, sem dependência de

\footnotetext{
${ }^{5}$ Cf. Disponível em: <https://news.microsoft.com/pt-br/banco-central-desenvolve-projeto-de-blockchain-comrecursos-do-microsoft-azure/> Acesso em: 23 de dez. 2018.

${ }^{6}$ Cf. Disponível em: <https://www.ccn.com/jpmorgans-ambitious-ethereum-based-blockchain-payments-trialadds-75-new-banks/> Acesso em: 23 de dez. 2018.
} 
um terceiro confiável (cartórios), além de garantir a privacidade e autotutela do cidadão na gestão dos seus dados pessoais"7.

Como um registro público irreversível e inalterável para documentos, contratos, propriedades e bens, podendo ser utilizado para conter informações e instruções, o bloco pode trazer inovadoras aplicações, como é o caso dos contratos inteligentes, tratados a seguir.

\section{SMART CONTRACTS}

Os smart contracts, ou contratos inteligentes, são uma combinação de algoritmos e códigos criados com o objetivo de aplicar os princípios comumente previstos em um ordenamento jurídico para o ciberespaço, destinando-se a formalizar e proteger relatórios em redes de computadores (BOMPREZZI, 2017, p. 20).

No contexto da blockchain, os contratos inteligentes significam transações que vão além da simples compra e venda de criptomoedas, podendo ter instruções mais extensas incorporadas a eles. Em uma definição mais formal, um contrato inteligente é um método de utilizar o bloco para criar um acordo entre determinadas partes (VIVALDI, 2016, p. 66).

A principal característica desses contratos é que eles, em regra, não usam intermediários que, como tal, tornariam mais caro a sua conclusão. Aos intermediários, incluem-se o aparato judicial e, portanto, o setor público, bem como os advogados que, em muitos casos, realizam uma atividade de pesquisa e negociação que leva à conclusão de um contrato conforme os interesses das partes (BOMPREZZI, 2017, p. 22).

Outro destaque é que tal contrato não pode ser modificado unilateralmente, dada a imutabilidade que caracteriza a blockchain, assim, uma vez lançado no bloco, o contrato é executado automaticamente quando as condições pré-estabelecidas são cumpridas, não havendo necessidade de contribuição humana, nem de um lado ou de outro (BOMPREZZI, 2017, p. 18).

\footnotetext{
${ }^{7} \mathrm{Cf}$. 
Como esses contratos possuem protocolos de computação que verificam e fazem respeitar a negociação ou execução de um contrato, possibilitam, assim, a desnecessidade de cláusulas contratuais, já que as mesmas podem ser parcialmente ou totalmente automatizadas, autorrealizável, fazendo com que tais contratos garantam uma maior segurança, apesar de tal entendimento ser aplicado em especial para à sua execução.

Em se tratando de execução, um destaque apontado em tal tecnologia é a sua auto execução, funcionando como um programa de computador, isto é, dada uma entrada, a mesma saída é sempre gerada: se $x$, então y. Para estabelecer se há condições para execução de um contrato, o sistema precisa receber informações do exterior, através dos chamados oráculos. Um oráculo ("oracle") é um terceiro, ou um banco de dados, ou uma pessoa humana a quem essa função foi atribuída, que fornece as entradas necessárias para o contrato inteligente (BOMPREZZI, 2017, p. 20).

O exemplo clássico usado para demonstrar o funcionamento dos contratos inteligentes é uma máquina que vende produtos de maneira automática, visto que, diferentemente de uma pessoa, tal máquina comporta-se por meio de algoritmos. Quando alguém insere o dinheiro e seleciona o que quer, a máquina libera o produto. Não há possibilidade de a máquina não respeitar o código, exceto se estiver com defeitos. Um contrato inteligente, da mesma forma, não deixa de executar o código prédefinido (VIVALDI, 2016, p. 67).

Os programas de computadores são de fato baseados na álgebra de Boole, na qual todos os valores são reduzidos a verdadeiro ou falso, em contraste com as regras normais de interpretação de contratos e seus critérios. O código não conhece ambiguidade, e as condições contratuais são claras desde o início. A rigidez do código e a auto execução garantem, em tese, a satisfação do cliente (VIVALDI, 2016, p. 21).

Dentre as discussões sobre o tema dos contratos inteligentes, encontra-se as implicações do direito dos contratos, uma vez que tal área é sempre dinâmica, evoluindo de acordo com as transformações da sociedade.

Conforme Carlos Roberto Gonçalves (2016, p. 974) "o contrato é uma espécie de negócio jurídico que depende, para sua formação, da participação de pelo menos duas 
partes". Ademais, sempre que o negócio jurídico "resultar de um mútuo consenso, de um encontro de duas vontades, estaremos diante de um contrato" (p. 275).

Por conseguinte, percebe-se que, conforme o artigo 422 do Código Civil Brasileiro de 2002, as partes necessitam de uma certa confiança na outra, visando resguardar "os princípios de probidade e boa-fé", a fim de que cada qual cumpra seu papel na obrigação.

Os contratos inteligentes incluem o mesmo tipo de acordo para fazer ou não fazer, mas eliminam, de modo geral, a necessidade de qualquer tipo de confiança entre as partes. Isso ocorre porque um contrato inteligente é definido pelo código ou executado e aplicado pelo algoritmo, automaticamente e sem critério (VIVALDI, 2016, p. 21).

Algumas dificuldades podem surgir por conta da imutabilidade unilateral do uso da blockchain, uma vez que, no momento em que o contrato é inserido, não pode mais haver, em regra, intervenção nele. Se, por um lado, isso pode ser positivo em termos de certeza de execução, por outro é problemático, se forem encontrados elementos que possam dar azo à invalidade ou rescisão do contrato, ou quando presentes circunstâncias que justifiquem uma retirada, renegociação ou rescisão (EENMAADIMITRIEVA; SCHMIDT-KESSEN, 2017, p. 26).

Nestes casos, a eficiência buscada através da execução automática de acordos, se levada a excesso, poderia levar ao efeito oposto. Por esta razão, alguns autores propuseram preferir a adoção de uma blockchain do tipo moderada, que mais facilmente permitem dar efeito a quaisquer medidas por parte de autoridade jurisdicional ou equivalente, como no caso da arbitragem, ou ainda a uma vontade alterada das partes, podendo materialmente extinguir um smart contract ou alterar o seu conteúdo (EENMAA-DIMITRIEVA; SCHMIDT-KESSEN, 2017, p. 25).

Como dito anteriormente, a auto execução de tal contrato tem o potencial de reduzir litígios no sistema jurídico tradicional, contudo, isso não significa, por outro lado, o total desaparecimento de causas judiciais. As partes podem ter que recorrer a um órgão para resolver conflitos, como, por exemplo, se um dos eventos do contrato ocorrer nulidade, anulação e etc. 
Além disso, embora se afirme que com a blockchain seja possível realizar contratos sem os chamados intermediários ou terceiros, novas figuras intervêm a esse respeito, como o programador de computação, das quais poderiam emergir os perfis de responsabilidade civil (BOMPREZZI, 2017, p. 27). O papel do programador de computador, ao qual se deve recorrer para criar um contrato inteligente, terá como finalidade a interpretação correta da vontade das partes e no qual estes depositarão sua confiança. Essa confiança, no entanto, poderia ser mal interpretada, já que o profissional pode dar vida a um contrato inteligente com resultados indesejáveis, por engano ou mesmo intencionalmente (CUCCURU apud BOMPREZZI, 2017, p. 27).

A rigidez do código ou protocolo, além disso, poderia forçar o profissional a simplificar as instruções recebidas, uma vez que o sistema binário busca abraçar inteiramente a complexidade da linguagem natural, composta de princípios gerais e cláusulas.

As acusações também podem ser feitas contra os responsáveis pela transmissão de informações fora do bloco, isto é, os oráculos, no caso de mau funcionamento ou adulteração. Além disso, o software usado para executar o contrato inteligente pode estar sujeito a defeitos ou bugs, o que poderia causar danos significativos aos usuários (SAVELYEV, 2017, p. 128).

Outro ponto que merece atenção é que uma parcela dos contratos inteligentes utilizam como bem de valor as criptomoedas, contudo, o ordenamento jurídico Brasileiro, bem como o de outros países, não possuem normas que consideram tal tecnologia como uma moeda válida. A exemplo disso, verifica-se que a Constituição Federal Brasileira de 1988, em seu artigo 21, inciso VII ${ }^{8}$, estabelece como competência da união a emissão de moedas, acrescentando-se, em seu artigo $164^{9}$, a exclusividade do Banco Central para tal tarefa.

A não consideração das moedas virtuais, amplamente difundidas pelo uso da blockchain, como uma moeda legal, traz incerteza a respeito de sua executividade como título de crédito, o que por consequência enfraquece a segurança jurídica dos negócios e contratos efetuados com o uso das mesmas.

\footnotetext{
${ }^{8}$ Art. 21. Compete à União: VII - emitir moeda.

${ }^{9}$ Art. 164. A competência da União para emitir moeda será exercida exclusivamente pelo banco central.
} 
Apesar de tais dificuldades, percebe-se que os smart contracts propõe diversos benefícios, tal como reduzir os custos mentais e os custos computacionais da transação, não apenas das partes do contrato, mas também de terceiros, bem como diminuir o número de casos judiciais, reduzindo os gastos públicos, visando uma descentralização gigantesca (VIVALDI, 2016, p. 66).

Apesar de tal proposta, a adoção da blockchain pode não resolver muitos dos aspectos críticos que foram relatados, existindo a possibilidade de sacrificar algumas formas de instituições de proteção dos direitos fundamentais, especialmente no campo contratual (BOMPREZZI, 2017, p. 33), demonstrando-se necessário a existência de instituições preparadas para lidar com os possíveis litígios advindos do uso dessa tecnologia.

O progresso tecnológico do bloco precisa de um certo grau de padronização e uma adoção generalizada, assim como um arcabouço jurídico ad hoc para lidar com os possíveis litígios, tal como a arbitragem, sendo provável que essa categoria de solução de conflito continuem sendo aplicada, adaptando as peculiaridades da tecnologia a estas, conforme exposto a seguir.

\section{ARBITRAGEM E CONFLITOS ENVOLVENDO A TECNOLOGIA BLOCKCHAIN E SMART CONTRACTS}

Em um conceito amplo, a arbitragem é "um meio célere de solução de controvérsias, realizada fora do Poder Judiciário e detentora de força executiva" (LIMA, 1998, p. 10). Tal meio pode ser utilizado em qualquer litígio que envolva matéria de direito patrimonial disponível, seja no Brasil ou em outro país (Idem).

Apesar de ser um dos mais antigos meios de composição de conflitos por um terceiro imparcial (SCAVONE JUNIOR, 2014, p. 16), a arbitragem pode ser considerada como um meio alternativo e adequado para a resolução de conflitos envolvendo a nova tecnologia blockchain, conforme exposto abaixo.

Inicialmente, verifica-se que diversas empresas que atualmente utilizam tal tecnologia nos mais diferentes campos, são as mesmas que utilizam, há tempos, a 
arbitragem para soluções de conflitos, como exemplos o Walmart ${ }^{10}$, o FedEx ${ }^{11}$ e a Microsoft ${ }^{12}$. Assim, parece intuitiva a progressão da arbitragem em disputas tradicionais para disputas que envolva a tecnologia da blockchain.

Outro fator a ser levado em consideração é o valor dado pela a arbitragem em relação à vontade das partes, seja na arbitragem institucional ou na ad hoc, sendo que naquela as partes possuem liberdade para escolher diferentes árbitros, bem como os procedimentos que deverão ser observador, submetendo a lide à uma determinada instituição, já nesta, as partes "escolhem um árbitro, e ambos disciplinarão, previamente, os procedimentos da arbitragem, específicos para o caso em questão" (LIMA, 1998, p. 12).

De qualquer maneira, a arbitragem prestigia o direito de escolha pelas partes, haja vista que ficam à sua disposição a escolha do árbitro. Além do mais, as partes podem inclusive utilizar um smart contract para estabelecer a forma em que desejam arbitrar.

Quanto à necessidade de conhecimento especializado para lidar com a blockchain e os contratos inteligentes, a arbitragem atende bem a tal ponto crítico, haja vista que as parte podem escolher árbitros com determinada especialização da matéria, assim, entende-se que tal escolha se dará por alguém que domine o assunto, acarretando que "o árbitro que decidirá o caso é um expert na matéria a ser solucionada" (SILVA, 2005, p. 147).

No judiciário, a solução por tais questões técnicas é realizada por meio de perícia "que, além do tempo que demanda, muitas vezes não conta com especialista de confiança das partes do ponto de vista técnico” (SCAVONE JUNIOR, 2014, p. 19). Logo, o árbitro que possui o conhecimento do assunto estaria também realizando a função que seria do perito no judiciário (SILVA, 2005, p. 147).

10 Cf. ROSSOW, Andrew. Why Walmart's Move To The Blockchain. Disponível em: <https://www.forbes.com/sites/andrewrossow/2018/09/25/why-walmarts-move-to-the-blockchain-could-domore-than-cure-e-coli-outbreaks/\#2b6d197f1100> Acesso em: 03 de jan. 2019.

11 Cf. MATHIS, Jack. FedEx is Testing Blockchain Tech. Disponível em: <https://www.ccn.com/tracking-keyshipments-fedex-is-testing-with-blockchain/> Acesso em: 03 de jan. 2019.

12 Cf. JUNCO, Pablo. Acelerando a adoção de blockchain pelas empresas. Disponível em: <https://news.microsoft.com/pt-br/acelerando-adocao-de-blockchain-pelas-empresas/> Acesso em: 03 de jan. 2019. 
Percebe-se que a arbitragem fornece flexibilidade às partes, posto que, diferentemente do sistema jurídico tradicional, o procedimento arbitral não é formal, podendo ser escolhido, além dos árbitros, o direito material e processual que serão utilizados (SCAVONE JUNIOR, 2014, p. 16), bem como os prazos e gastos que "são previamente definidos e acordados pelas partes, sem burocracia e os diversos problemas da justiça estatal" (LIMA, 1998, p. 12).

Por consequência, há uma resolução de conflito de forma mais célere, sendo menos provável que isso impeça a velocidade com que a tecnologia do bloco costuma funcionar.

O tempo nas transações envolvendo a tecnologia blockchain e os smart contracts não condiz com o tempo decorrido em processos judicias, sendo a celeridade na resolução do litígio algo que se faz extremamente necessária. A arbitragem tem o potencial de ser o meio procurado para que encontre soluções no tempo real das transações, posto que "as custas judicias são menos preocupantes do que a demora na prestação jurisdicional, insuportável e insustentável" (SILVA, 2005, p. 149) para esses litigantes.

Um outro ponto que merece destaque é a Convenção de Arbitragem de Nova lorque, bem como o reconhecimento de sentenças arbitrais estrangeiras e sua execução.

Criada em 10 de junho de 1958, foi um marco da arbitragem em nível mundial. Ela possui dezesseis artigos que regulam normas para "reconhecimento e execução de sentenças arbitrais" (SILVA, 2005, p. 59).

A convenção permite que uma parte busque a execução da sentença em qualquer país que tenha assinado a convenção. Atualmente, existem 159 países signatários ${ }^{13}$.

O artigo II da Convenção de Nova lorque estabelece que a sentença arbitral é aquela proferida por um árbitro ou por órgãos arbitrais permanentes. O artigo III também destaca que:

\footnotetext{
${ }^{13}$ Cf. Contracting States - New York Arbitration Convention. Disponível em: <http://www.newyorkconvention.org/countries> Acesso em: 03 de jan. 2019.
} 
Cada Estado signatário reconhecerá as sentenças como obrigatórias e as executará em conformidade com as regras de procedimento do território no qual a sentença é invocada, de acordo com as condições estabelecidas nos artigos que se seguem. Para fins de reconhecimento ou de execução das sentenças arbitrais às quais a presente Convenção se aplica, não serão impostas condições substancialmente mais onerosas ou taxas ou cobranças mais altas do que as impostas para 0 reconhecimento ou a execução de sentenças arbitrais domésticas.

De fato, a tecnologia blockchain alcança milhares de participantes da rede de computadores em diferentes países, assim, sem dúvida a aplicação da arbitragem e o reconhecimento de sentenças estrangeiras e sua execução, se mostra um recurso promissor para garantir que a resolução de litígios possa ser amplamente aplicado mundialmente.

\section{CONSIDERAÇÕES FINAIS}

Nos últimos anos, o smart contract e a blockchain tem recebido um destaque crescente e, por consequência disto, a exploração para entender o seu funcionamento tem aumentado cada vez mais.

Os desafios apresentados pelo uso de tais tecnologias tem demonstrado que 0 modo como é solucionado os conflitos atualmente, em especial pelo método jurídico tradicional, possui certas desvantagens em lidar com algo completamente novo e diferente, que por suas características não se adequam a conceitos jurídicos habituais, bem com inviabiliza o uso do sistema habitual para soluções destes novos litígios. Este ponto traz à tona a escolha da arbitragem como meio alternativo e adequado para solucioná-los, haja vista seus flexíveis aspectos em lidar com tal problemática.

Existem diversas perspectivas e expectações sobre o que tal tecnologia causará no futuro próximo, contudo, variados pontos necessitam serem desdobrados e aperfeiçoados para que o seu uso se consolide em uma maior seguridade e efetividade, seja no campo do direito, da economia ou no próprio meio técnico.

A tecnologia blockchain tem chances de mudar a maneira de fazer negócios comumente conhecido. À medida que a tecnologia evolui, fica claro que a arbitragem 
tem o potencial de desempenhar um papel crítico. Com essa nova tecnologia, necessária se faz a familiarização entre árbitros e operadores do Direito, compreendendo o seu processo, benefícios e possíveis armadilhas, aprimorando as habilidades e a capacidade de adaptação a mudanças contínuas, além de se preparar para tornar-se parte integrante dessa nova e desafiadora fronteira.

\section{REFERÊNCIAS}

BOMPREZZI, Chantal. Blockchain e assicurazione: opportunità e nuove sfide. Rivista Diritto Mercato Tecnologia - Nuova Editrice Universitaria, Roma, pp. 37, Anno VII, Luglio 2017.2 Disponível em: <https://www.dimt.it/images/pdf/Blockchaineassicurazione.pdf>. Acesso em: $15 \mathrm{de} \mathrm{fev.}$ de 2019.

BITCOIN. Bitcoin: A Peer-to-Peer Electronic Cash System. Disponível em: $<$ https://bitcoin.org/bitcoin.pdf> Acesso em: 13 de jan. 2018.

BRASIL. Constituição Federal. Brasília: Senado Federal, 1988. Disponível em: <http://www.planalto.gov.br/ccivil_03/Constituicao/Constituicao.htm>. Acesso em: $15 \mathrm{de}$ jan. de 2019.

BURRICCO, Edoardo. Le evoluzioni della moneta virtuale prospettive ed incognite legate ai bitcoin. pp. 97, Roma, 2017. Tesi di Laurea in Diritto dei mercati e degli intermediari finanziari. Universtà LUISS Gido Carli, relatore Mirella Pellegrini. Disponível em: <https://tesi.luiss.it/19767/1/669041_BURRICCO_EDOARDO.pdf>. Acesso em: 13 de fev. de 2019.

CAPOGNA A., PERAINO L. et. al., Bitcoin: profili giuridici e comparatistici. Analisi e sviluppi futuri di un fenomeno in evoluzione. Rivista Diritto Mercato Tecnologia, Roma, Anno V, Numero 3, pp. 32-74, Luglio/Settembre 2015. Disponível em: < https://www.dimt.it/wp-content/uploads/2016/06/Contributo-di-Alexandro-CapognaLeandro-Peraino-Silvia-Perugi-Marco-Cecili-Giovanni-Zborowski-Andrea-Ruffo.pdf>. Acesso em: 15 de fev. de 2019.

EENMAA-DIMITRIEVA, Helen; SCHMIDT-KESSEN, Maria José. Regulation Through Code as a Safeguard for Implementing Smart Contracts in No-Trust Environments. European University Institute - Department of Law Research Paper, No. 2017/13, ISSN 1725-6739. Disponível em: <http://cadmus.eui.eu/bitstream/handle/1814/47545/LAW_2017_13.pdf?sequence=1\&is Allowed=y>. Acesso em: 14 de fev. de 2019. 
FedEx is Testing Blockchain Tech. Disponível em: $<$ https://www.ccn.com/tracking-key-shipments-fedex-is-testing-with-blockchain/> Acesso em: 03 de jan. 2019.

GONÇALVES, Carlos Roberto. Direito Civil Esquematizado: parte geral : obrigações e contratos ; coordenador Pedro Lenza. - 6. ed. - São Paulo : Saraiva, 2016.

. JPMorgan's Ethereum-Based Blockchain Payments Trial Adds 75 Banks. Disponível em: <https://www.ccn.com/jpmorgans-ambitious-ethereum-basedblockchain-payments-trial-adds-75-new-banks/> Acesso em: 23 de dez. 2018.

LIMA, Alex Oliveira Rodrigues de, - Arbitragem: um novo campo de trabalho. 2.a ed. rev. e ampl. - São Paulo: llgu, 1998.

LÓPEZ, Alejandro. Implicaciones jurídicas del uso del bitcoin en Colombia. Validez del contrato de compraventa comercial con bitcoins. San Juan de Pasto. Project Report Universidad de Nariño, pp. 187, 2015. Disponível em: < http://biblioteca.udenar.edu.co:8085/atenea/biblioteca/90756.pdf>. Acesso em: 15 de fev. de 2019.

MICROSOFT NEWS CENTER BRASIL. Acelerando a adoção de blockchain pelas empresas. Disponível em: <https://news.microsoft.com/pt-br/acelerando-adocao-deblockchain-pelas-empresas/> Acesso em: 03 de jan. 2019.

SAVELYEV, Alexander. Contract Law 2.0: 'Smart' Contracts as the beginning of the end of classic contract law. Information \& Communications Technology Law, pp. 116134, Vol. 26, NO. 2, 2017, ISSN: 1469-8404. Disponível em: < http://dx.doi.org/10.1080/13600834.2017.1301036>. Acesso em: 13 de fev. de 2019.

SCAVONE JUNIOR, Luiz Antonio. Manual de Arbitragem. 5 ed. rev., atual. e ampl. Rio de Janeiro: Forense, 2014.

SILVA, Adriana dos Santos. Acesso à justiça e arbitragem: um caminho para a crise do judiciário - Barueri, SP: Manole, 2005.

VIVALDI, Ilaria. Blockchain: la rivoluzone digitale nel mondo finanziario. Tesi di Laurea Magistrale in Finanza Aziendale e Mercati Finanziari, universtà di Pis, relatore Riccardo Cambini. pp. 112, Pisa, 2015/2016. Disponível em: <https://etd.adm.unipi.it/t/etd-06012016-164829/>. Acesso em: 15 de fev. de 2019.

Why Walmart's Move To The Blockchain. Disponível em: <https://www.forbes.com/sites/andrewrossow/2018/09/25/why-walmarts-move-to-theblockchain-could-do-more-than-cure-e-coli-outbreaks/\#2b6d197f1100> Acesso em: 03 de jan. 2019. 
ZEN, Alberto. Bitcoin: Analisi Tecnica ed Economica. Tesi di Laurea in Amministrazione Finanza e Controllo, Università Ca'Foscari Venezia, relatore Marco Corazza, pp. 108, Venezia, 2015. Disponível em: <http://dspace.unive.it/bitstream/handle/10579/6740/821411-1184046.pdf>. Acesso em: 15 de fev. de 2019. 